\title{
Discussion of Session II
}

\author{
Chairman: Professor E. Lebacq
}

Dr A. J. Karlish (Central Chest Clinic, Battle Hospital, Reading, Berkshire): I would like to comment on the use of bronchoscopy in the diagnosis of sarcoidosis. We have done eighty-two bronchoscopies in sarcoid patients and of these thirty-two were classified as positive and five as probably positive, a total of $45 \%$. I would dispute that you cannot find tubercle bacilli microscopically in non-caseating tuberculous lesions. We have done pleural biopsies in about 120 cases of tuberculous pleural effusions. Since the majority were early cases there was no caseation or perhaps minimal caseation in $40 \%$ of the cases. AFB were found microscopically in two thirds of the cases with caseation and about half of those without caseation. It is true that the search can be laborious and on occasions hundreds of sections were examined, but the search for AFB in non-caseating tuberculosis is not unrewarding.

DR R. DRURY: May I ask if pleural biopsies were positive, was that of the fluid or of the section?

KARLISH: Well, the pleural biopsies showed histology which corresponded to what is described as "noncaseating tubercle', namely there was a trace or hardly any caseation and such tubercles could very easily be mistaken for those occurring in what we call sarcoidosis and yet on section we found $50 \%$ tubercle bacilli. I do not know whether this answers your question or not.

DRURY: I think it does answer the question. The point I was trying to make is that we do not find AFB in tuberculous lesions of pleura very frequently, though these are commonly, in our experience, seen in caseous pleura. Secondly, one does not find tubercle bacilli in the fluid at the same time as your pleural biopsy rather than in the section which is small.

KARLISH: Yes, this is a different matter. The culture fluid is positive only in $30-35 \%$ of the cases; the pleural biopsy is positive in about $80 \%$ of the cases, so there is a vast difference.

DR W. Jones Williams (Institute of Pathology, Cardiff Royal Infirmary): I must compliment Dr Drury on his beautiful presentation and on his nice diagrams. However, I must disagree with one or two things in those diagrams. I am not at all happy about this idea that the Kveim test of sarcoid granuloma goes through a stage of foreign body granuloma before it becomes a sarcoid lesion, in that if one finds foreign material in a Kveim test it is much safer to say that this is a foreign body reaction and not a positive Kveim test. However, you can obviously occasionally find foreign material and definite granulomas and if you find separate granulomas with no foreign material call it 'positive', but if you find only a few granulomas and they all contain foreign material it is very important to call that 'negative'. The second point $I$ would make very briefly is about the change in the collagen and this word 'necrosis'. If one looks at this under electron microscopy, with replication methods, where you coat the material and look at it under the electron microscope, you can see that there is very little actual necrosis, it is made up of fibrin and early collagen and reticulin. I am not at all sure if this is actual necrosis, though on the $\mathrm{H} \& \mathrm{E}$ section it looks like it.

SCADDING: I only just want to make one comment here, to draw your attention to the fact that in all this discussion the words 'positive' and 'negative' are being used in quite different senses. When we discuss, say, tubercle bacilli, 'positive' means tubercle bacilli were found; negative means that they were not found-it does not necessarily mean they were not there but it means they were not found. Whereas 'positive' and 'negative' in relation to histology, as far as I can make out, is simply a statement of opinion of the pathologist that the appearances he saw should be classified in a certain way. These are quite different meanings, although we use the same words to describe the two. This is not a criticism, it is just the way people use words but it is important that we should bear in mind that these two usages of the same words seem to refer to similar facts, but in fact refer to very different sorts of facts.

I sprang up first really because I wanted just to ask a question of Dr Rees whilst it is still in my mind. He mentioned at the end that among the patients who were investigated in Singapore, there was a group of seven or eight whose leprosy, whatever type it was, was considered to be active but I do not think he told us whether there was any difference in the Kveim test results in those from that in the general series.

DR R. J. W. REES (National Institute for Medical Research, Mill Hill, London): One positive lepromin, one positive Kveim reaction and of the equivocals-if you take the one positive-five of the seven occurred in patients who had had 2 or more years' treatment.

KARLISH: I would like to take up one or two points which were mentioned both by Dr Mikhail and by Dr Drury. First of all, may I show you the results of Kveim tests done in 600 patients in Reading. The percentage of so-called 'false positives' in 186 controls is $3 \%$, more or less in keeping with what has been previously published, but three of the five 'false positive' reactions were in a group of fifty-five tuberculous patients, a disturbing finding since such cases may pose problems in differential diagnosis. Two of the three tuberculous patients were Pakistanis with florid glandular tuberculosis.

Table 1. Kveim tests in sarcoidosis: 600 patients

\begin{tabular}{lccc}
\hline & $\begin{array}{l}\text { Number of } \\
\text { patients }\end{array}$ & $\begin{array}{l}\text { Number } \\
\text { positive }\end{array}$ & $\%$ Positive \\
\hline Sarcoidosis confirmed & 128 & 83 & $65 \%$ \\
Sarcoid suspects & 286 & 139 & $49 \%$ \\
Controls & 186 & 5 & $3 \%$ \\
\hline
\end{tabular}

